\title{
Estudio observacional de los pacientes ingresados en un Servicio de Medicina Interna
}

\author{
J. L. DELGADO MORALES, R. ALONSO DEL BUSTO, I. PASCUAL CALLEJA, \\ $M^{a}$ M. VILLACORTA MARTÍN, P. ERGUETA MARTÍN, E. GONZÁLEZ \\ SARMIENTO
}

Servicio de Medicina Interna. Hospital Universitario. Valladolid

OBSERVATIONAL STUDY OF IN-PATIENS IN AN INTERNAL MEDICINE DEPARTMENT

\section{RESUMEN}

Objetivos: Analizar las patologías más frecuentes ingresadas en un servicio de Medicina Interna, los motivos de ingreso hospitalario, los diagnósticos al alta (según el síntoma guía de ingreso), el grupo de población afectada y su correlación entre ellos.

Pacientes y métodos: Sobre un total de 758 ingresos en Medicina Interna del Hospital Universitario de Valladolid, durante el año 1999, en función de los informes de alta e historias clínicas, se realizó un estudio epidemiológico descriptivo observacional, utilizando las variables de sexo, edad, motivo de ingreso (síntoma guía) y diagnósticos al alta. Los resultados obtenidos fueron representados por medio de diagramas de sectores y de barras, en función de las variables analizadas. Para la síntesis de datos fueron utilizadas, medidas de tendencia central y de dispersión. El estudio estadístico empleado fue el programa SPSS versión 10.0 para Windows. El análisis no paramétrico para muestras independientes fue realizado con: la prueba de la mediana y U de Mann-Whitney, y el análisis paramétrico con el test $\chi^{2}$ y contraste de Kolmogorov-Smirnov.

Resultados: La mediana de edad es de 70 años, rango 84 años, rango intercuartílico de 23 , con una moda en varones de 75 años y en mujeres de 86 años. La distribución por sexo: varones $51 \%$, mujeres $49 \%$. Los motivos de ingreso más frecuentes son: la disnea $(35 \%)$ y la focalidad Neurológica $(11 \%)$. Los diagnósticos al alta más frecuentes: EPOC reagudizado por infección respiratoria (11\%), neumonía (8\%) y ACVA isquémico $(7 \%)$.

Conclusiones: Los pacientes ingresados en este servicio son de edad avanzada (sobretodo en mujeres), $(\alpha=0,05)$ significación estadística. La edad no sigue una distribución normal $(\alpha=0,05)$ significación estadística. La frecuencia en la distribución por sexo es similar. El motivo de ingreso más frecuente es la disnea (35\%). Los diagnósticos al alta más frecuentes son: La EPOC (11\%), la neumonía (8\%) y el ACVA isquémico $(7 \%)$. La prevención $1^{\mathrm{a}}$ y $2^{\mathrm{a}}$ y una mejora de la medidas terapéuticas de las enfermedades crónicas cardiorrespiratorias, reduciría, significativamente la presión asistencial de los servicios de Medicina Interna y mejoraría la calidad de vida de la población, dado que estamos frente a enfermedades que están dentro de las cuatro primeras causas de mortalidad en el mundo.

PALABRAS CLAVE: Estudio observacional. Pacientes ingresados. Medicina Interna.
ABSTRACT

Objectives: To analyse the entered more frequent diseases in an Internal Medicine Department, the reasons for hospital admission diagnosis at discharge (according to entrance symptom's guide), the group of affected population and its correlation among them.

Patients and methods: Over a total of 758 internal medicine admissions of the University Hospital of Valladolid during the year 1999 based on the information of discharge and clinical histories, a descriptive and observational epidemic study was made using the variables of sex, age, reason for admission (guide symptom) and diagnosis at discharge. The obtained results were represented by mean of diagrams of sectors and bars according to the analyzed variables. The data synthesis was made by measures of central tendency and dispersion. SPSS 10.0 version for windows program was used for the statistical study. The non parametric analysis for independent samples was made by the test of median and the U of Mann Whitney, and the parametric by chi-squired test and resistance of Kolmogorov-Smirnov.

Results: The median of age is 70 years. Rank 84 years. Interquartile rank 23, fashion in men 75 years and in women 86. The distribution in sex men 51\%, women 49\%.The more frequent reasons for entrance are dyspnea (35\%) and neurological focus (11\%).The more frequent diagnosis at discharge are dyspnea and chronic obstructive lung disease worsened by respiratory infection (11\%), pneumonia (8\%) and acute isquemic stroke $(7 \%)$.

Conclusions: In-patiens in this service, are advanced in years (mainly women) ( $\alpha=0.05)$. The age does not get a normal distribution $(\alpha=0.05)$. The frequency of the distribution in sex is similar. The most frequent reason for admission is dyspnea (35\%). The most frequent diagnoses at discharge are chronic obstructive lung disease (11\%), pneumonia $(8 \%)$ and acute ischemic stroke (7\%). The primary and secondary prevention and an improvement of the therapeutic measures of chronic cardiopulmonary disease would reduce significantly the welfare pressure in Internal Medicine Departement and they would improve the population's life quality given that we are opposed to the diseases which are among the four first mortality causes in the world.

KEY WORDS: Observational study. Patients. Internal Medicine.

Delgado Morales JL, Alonso del Busto R, Pascual Calleja I, Villacorta Martín MáM, Ergueta Martín P, González Sarmiento E. Estudio observacional de los pacientes ingresados en un Servicio de Medicina Interna. An Med Interna (Madrid) 2004; 21: 3-6.

Trabajo aceptado: 16 de junio de 2003 


\section{INTRODUCCIÓN}

La observación en la clínica diaria del aumento progresivo de pacientes con edades avanzadas (1-3) y enfermedades crónicas asociadas $(1,3)$, ha motivado la realización de este estudio. Los reingresos de este tipo de pacientes, parece ser, que están en función de la edad y de las patologías asociadas que presentan, a mayor edad y con más patología asociada más reingresos hospitalarios $(1,3)$.

Para unificar criterios a la hora de clasificar las enfermedades y calcular los costes, surgieron los GRD's ( grupos relacionados por el diagnóstico). El motivo inicial de ellos fue diseñar una estructura adecuada para analizar la asistencia médica y la utilización de los servicios en el entorno hospitalario (4). En los GRD's el diagnóstico principal al alta viene definido por el síntoma guía clínico que motivó su ingreso hospitalario.

Según ello, los objetivos de este estudio fueron: el análisis de las patologías más frecuentes ingresadas en un Servicio de Medicina Interna del Hospital Clínico de Valladolid, el tipo de población afectada, los diagnósticos al alta (según el síntoma guía que motivó el ingreso) y la correlación de todos estos parámetros entre sí. En función de estas premisas se intentó definir hacia donde va encaminada la labor asistencial dentro de la Medicina Interna.

\section{MATERIAL Y MÉTODO}

Fueron revisados 758 ingresos en dicho servicio de Medicina Interna, durante el año 1999, analizando los informes de alta e historias clínicas. Las variables analizadas fueron el sexo, la edad, el motivo de ingreso hospitalario (entendido como el síntoma guía principal que requiere el ingreso) y el diagnóstico al alta, según los GRD’s.

En este estudio epidemiológico observacional retrospectivo, la síntesis de algunos datos fue realizada mediante, medidas de tendencia central y medidas de dispersión. La representación de resultados fue mediante diagramas de sectores y diagramas de barras, en función de las variables estudiadas.

Para el estudio se aplicó el programa SPSS versión 10.0 para Windows. En el análisis no paramétrico de muestras independientes fueron aplicadas la prueba de la Mediana y U de Mann whitney, para el análisis paramétrico el test $\chi^{2}$ y el contraste de Kolmogorov-Smirnov.

\section{RESULTADOS}

La edad media de los pacientes en ambos sexos es de $66 \pm 14$ años, la mediana de 70 años, rango de 84 años, rango intercuartílico 23, la moda de 73 años, moda en varones de 75 años, y moda en mujeres de 86 años (Tablas I y II).

El contraste de normalidad (prueba de Kolmogorov-Smirnov) para la variable edad, no seguía una distribución normal (Fig. 1).

La mediana de edad de ingreso en hombres no fue igual a la de las mujeres para el estadístico $\chi^{2}$, con corrección por continuidad de Yates, $\mathrm{p}<0,05$ existían diferencias significativas entre ambas medianas.

La prueba de Mann-Whitney que contrasta si existen diferencias significativas en cuanto a la edad de los hombres y de
TABLA I

SÍNTESIS DE DATOS

Media: $66 \pm 14$ años

Mediana: 70 años

Rango: 84 años

Moda: 73 años

Moda varones: 75 años

Moda mujeres: 86

Rango intercuartílico: 23

TABLA II

SÍNTESIS DE DATOS POR SEXO

\begin{tabular}{ll}
\hline Varón & Mujer \\
\hline Media: 65 años & Media: 67 años \\
Mediana: 69 años & Mediana: 72 años \\
Moda: 75 años & Moda: 86 años \\
Rango: 84 años & Rango: 82 años \\
Rango intercuartílico: 20,75 & Rango intercuartílico: 26 \\
\hline
\end{tabular}

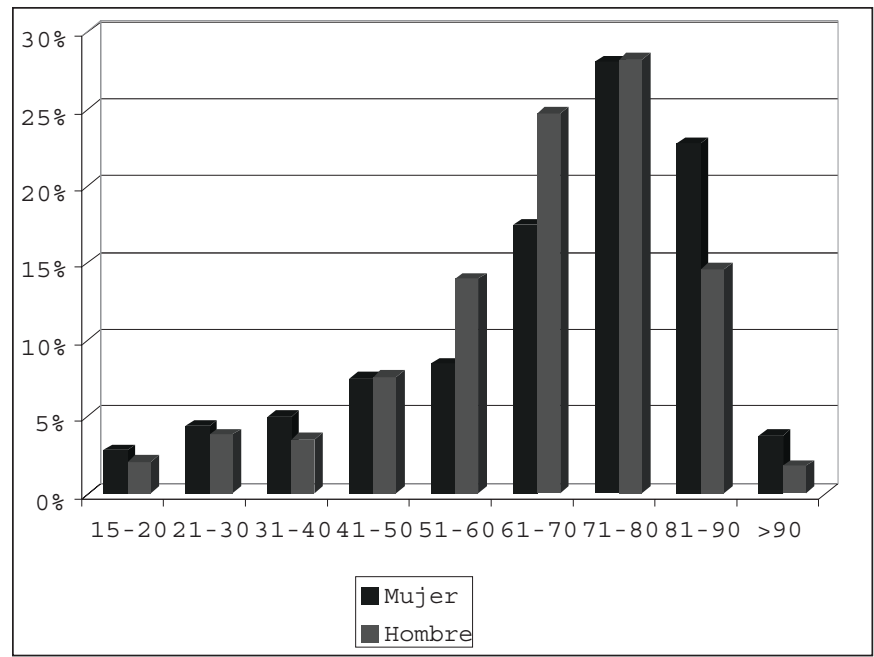

Fig. 1. Distribución por sexo-edad.

las mujeres ingresadas, verificó que los pacientes que ingresan en este servicio de Medicina Interna son de edades avanzadas ( $\mathrm{p}<0,05)$, sobre todo en mujeres alcanzando niveles de significación estadística $(\alpha=0,05)$ (Tablas I y II, Fig. 1$)$.

El porcentaje de ingresos en cuanto al sexo es similar en esta serie (49\% mujeres, 51\% varones). Sin embargo de los 15 a los 40 años predominaron levemente los ingresos de mujeres, de los 51 a los 70 años aumentaron varones, de los 71 a los 80 años fue similar en los dos sexos y a partir de los 81 años es predominante en la mujer (Fig. 1).

El motivo de ingreso de estos pacientes, en función del síntoma guía en el servicio de urgencias, (independientemente de sus enfermedades de base), fue la disnea con un $35 \%$ de los casos. Es por consiguiente el síntoma más frecuente por el que los pacientes ingresan. La focalidad neurológica ocupa el segundo lugar con un $11 \%$ (Fig. 2).

En el varón los motivos de ingreso mas frecuentes fueron, la rectorragia, la epigastralgia, el dolor pleurítico y la focali- 


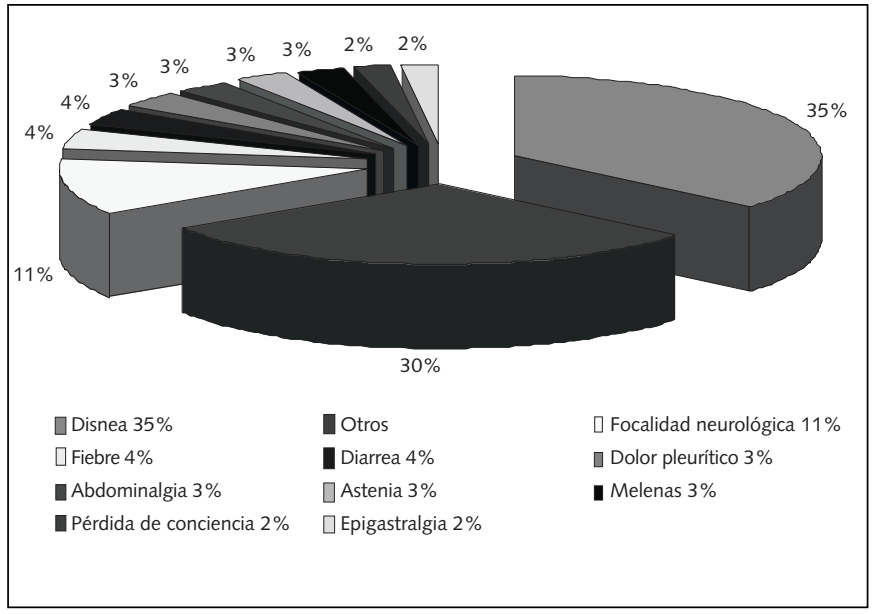

Fig. 2. Motivos de ingreso.

dad neurológica. En la mujer fueron la lumbalgia, la astenia, la diarrea y la disnea. Esta relación (sexo del paciente y motivo de ingreso) no alcanzó niveles de significación estadística, $\mathrm{p}>0,05$.

Los diagnósticos al alta más frecuentes, en general fueron la EPOC reagudizada por infección respiratoria $11 \%$, la neumonía 8\%, y el ACVA isquémico 7\% (Fig. 3).

En los diagnósticos al alta por sexo, en varón fueron más frecuentes la EPOC, la neumonía y la hemorragia digestiva alta. En la mujer sin embargo predominó más la patología asmática, la insuficiencia cardiaca y la anemia ferropénica. Esta relación (sexo del paciente y diagnóstico al alta), no fue muy fuerte, pero si estadísticamente significativa $(\mathrm{p}<0,05)$ (Fig. 4).

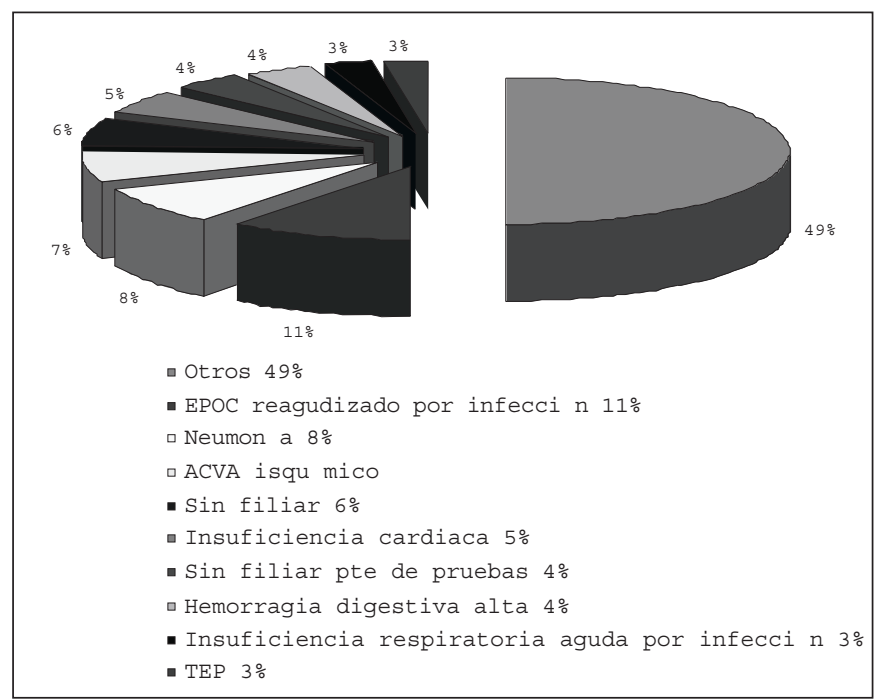

Fig. 3. Diagnóstico al alta.

\section{DISCUSIÓN}

Los resultados de este estudio reflejan que en el servicio de Medicina Interna del Hospital Universitario de Valladolid, el porcentaje de ingresos es similar en el varón y en la mujer.

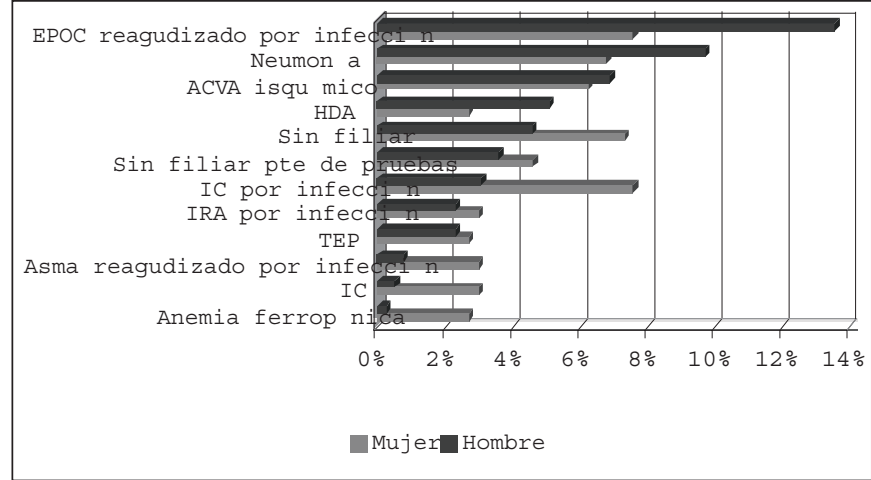

Fig. 4. Diagnóstico al alta por sexo.

Los pacientes son de edades avanzadas. En la variable edad fue necesario el cálculo de mediana, la moda y el rango, porque, como refleja la figura 2 , no sigue una distribución normal. La media, nos daría una información parcialmente sesgada de la edad de los pacientes. El cálculo de la mediana, y la moda como medidas de tendencia central y el rango como medida de dispersión, constata que la población estudiada es de edad avanzada, sobre todo en mujeres.

La disnea y la focalidad neurológica son los motivos de ingreso mas frecuentes, ambas patologías representan casi la mitad de los ingresos de Medicina Interna en esta serie.

La patología cardiorrespiratoria ocupan un lugar primordial. La EPOC, la Neumonía, el ACVA isquémico y la insuficiencia cardiaca, son los diagnósticos al alta más frecuentes.

Probablemente debido a la edad avanzada de los pacientes y a que el varón es ya en sí mismo un factor de riesgo de padecer insuficiencia cardiaca y fallece antes, la mujer ocupa un lugar importante en la insuficiencia cardiaca en nuestra serie.

Teniendo en cuenta todo lo anterior la prevención $1^{\mathrm{a}}$ y $2^{\mathrm{a}}$ de este tipo de patologías reduciría la presión asistencial en los servicios de Medicina Interna hospitalarios y mejoraría la calidad de vida de los pacientes.

La actuación sobre los factores de riesgo, sobretodo los factores de riesgo cardiovascular, es uno de los objetivos primordiales que deberían de plantearse según este estudio, y otros estudios encontrados en la literatura $(5,6)$. Esto no significa que hasta ahora no se hayan tomado medidas sobre los factores de riesgo, si no que estas, en función de lo reflejado en este estudio no son suficientes. Si bien es verdad que la edad es un marcador de riesgo, endógeno a la persona, no controlable, que aumenta la probabilidad de enfermar y no es responsable directo de producir enfermedad.

El informe GOLD (Global Iniciative For Chronic Obstuctive Lung Disease), destaca que la EPOC, es actualmente la cuarta causa de mortalidad en el mundo, después de la insuficiencia cardiaca, el cáncer y el infarto agudo de miocardio. La EPOC, cada año acaba con la vida de 2,5 millones de personas. Este informe pone de manifiesto la necesidad de actuar sobre los factores de riesgo de esta enfermedad, y apunta las directrices de tratamiento de la misma (7).

Creemos que la labor del médico debe ir encaminada en mejorar la prevención primaria, secundaria y puesta al día de la terapéutica de estas patologías, tanto a nivel hospitalario como del médico de Atención Primaria. Sería de interés fomentar, lugares de encuentro multidisciplinarios, mesas redondas y talleres educativos para conseguir estos fines. 


\section{Bibliografía}

1. Viana A, Carballo F, Beato I, et al. An análisis of 14.000 patients admitted to an internal medicine service during 1982-1988. An Med Interna (Madrid) 1990; 7(9): 459-62.

2. Álvares E, Sachicumbi A, Franco AS, Saavedra J. Pneumonia in an internal medicine service. Acta Med Port 1996; 9 (2-3): 79-85.

3. Chirstensen SB, Gjorup T. Admission pattern at a department of internal medicine. Factors of significance for readmission within three months after discharge. Ugeskr Laeger 1998; 160 (16): 2396400 .

4. Richard F Averill, Norbert Goldfield, Barbara A, SteinbecK. Grupos relacionados por el diagnóstico (GRD's), 1995. Health Information Systems, 3M-SIGESA,
5. He J, Ogden LG, Bazzano LA, et al. Risk factors for congestive heart failure in US men an women: NHANES I epidemiologic study. Arch Intern Med 2001; 161 (7): 996-1002.

6. Quereshi AI, Suri MF, Guterman LR, Hopkins LN. Ineffective secondary prenvention in surviviors of cardiovascular events in the US population: report from the Third National Health and Nutrition Examination. Arch Intern Med 2001; 161 (13): 1621-8.

7. Pauwels RA, Buist AS, Calverley PMA, Jenkins CR, Hurd SS. Global Strategy for the Diagnosis, Management, and Prevention of Chronic Obstructive Pulmonary Disease . NHLBI/WHO Global Initiative for Chronic Obstructive Lung Disease (GOLD) Workshop Summary. Am J Respir Crit Care Med 2001; 163: 1256-1276. 